\title{
Study of the Solar Modulation of Local Interstellar Protons with AMS-02, PAMELA, Neutron Monitors and Voyager 1
}

\author{
Claudio Corti* \\ Physics and Astronomy Department, University of Hawaii at Manoa, 96822, HI, US \\ E-mail: corti@hawaii.edu \\ Veronica Bindi \\ Physics and Astronomy Department, University of Hawaii at Manoa, 96822, HI, US \\ E-mail: bindi@hawaii.edu \\ Cristina Consolandi \\ Physics and Astronomy Department, University of Hawaii at Manoa, 96822, HI, US \\ E-mail: cconsola@hawaii.edu

\section{Julia Hoffman} \\ Physics and Astronomy Department, University of Hawaii at Manoa, 96822, HI, US \\ E-mail: kurowska@cern.ch
}

\section{Kathryn Whitman}

Physics and Astronomy Department, University of Hawaii at Manoa, 96822, HI, US

E-mail: kwhitmanehawaii.edu

Galactic cosmic rays (GCRs) entering the heliosphere are disturbed by the magnetic field of the Sun, which varies with a period of 11 years. The solar modulation affects the GCR fluxes up to few tens of $\mathrm{GeV}$, modifying the shape and the intensity of the local interstellar spectrum (LIS). The time variation of the galactic cosmic protons at Earth can be studied indirectly on ground with the neutron monitors (NMs) and directly from space with AMS-02 (2011-now) and PAMELA (20062010). A new parametrization of the LIS will be presented, based on the latest data from AMS02 and Voyager 1. Using the framework of the force-field approximation, the solar modulation parameter will be extracted from the time-dependent proton fluxes measured by PAMELA. The results will be compared with the modulation parameter inferred by NMs.

The 34th International Cosmic Ray Conference,

30 July- 6 August, 2015

The Hague, The Netherlands

\footnotetext{
*Speaker.
} 


\section{Introduction}

Galactic cosmic rays (GCRs) up to PeV scale are thought to be produced and accelerated in supernovae explosions within our galaxy. During their travel to Earth, they interact with the interstellar gas and the galactic magnetic field, and by the time they reach the boundary of the heliosphere, their spectrum is substantially different from the injection one and is called local interstellar spectrum (LIS). The LIS is in turn affected by the heliospheric magnetic field (HMF), which varies with time: the solar modulation modifies the GCR fluxes up to few tens of GeV, changing both the shape and the intensity of the LIS.

The equation governing the propagation of GCRs in the heliosphere is the Parker equation [1]; Gleeson and Axford [2] derived an approximate solution, so called force-field approximation (FFA) , which, due to its simplicity, is still being used nowadays, despite the availability of numerical models. According to the FFA, the GCR flux $J$ measured at Earth at the time $t$ is related to the LIS $J_{L I S}$ with the formula

$$
J\left(1 \mathrm{AU}, t, E_{k}\right)=J_{L I S}\left(E_{k}+\Phi(t)\right) \frac{E_{k}\left(E_{k}+2 M\right)}{\left(E_{k}+\Phi(t)\right)\left(E_{k}+\Phi(t)+2 M\right)}
$$

where $E_{k}$ is the kinetic energy, $M$ the GCR mass and $\Phi=Z e \phi$ is the solar modulation parameter, with $Z$ the GCR charge number, $e$ the elementary charge and $\phi$ (sometimes called itself solar modulation parameter) has the units of an electric potential. The interpretation of $\Phi$ is straightforward: it is the average energy loss experienced by GCRs from the boundary of the heliosphere up to the Earth.

The solar modulation has two main cycles: the most important one is related to the 11-year periodic solar activity, while the other is related to the 22-year periodic reversal of HMF occurring at each peak of solar activity. These cycles can be measured directly from Earth by looking at the sunspot number and their effect on GCRs is continuously measured on ground since the 50's with the world network of neutron monitors (NM), which count the number of neutrons produced by the interactions of GCRs with the atmosphere. NM are integrating devices, meaning that what is actually measured is the integral of the GCR flux above the rigidity cutoff pertaining to the NM location. For this reason, the extraction of the solar modulation parameter from their data depends on the assumed shape of the LIS. Balloon- and space-borne experiments (such as BESS [3], PAMELA [4], AMS-02 [5]) are instead able to measure the energy dependence of the GCR flux, therefore providing a way to disentangle the degeneracy between $\Phi$ and $J_{L I S}$. In 2006, the PAMELA experiment was launched aboard a satellite in low Earth orbit and since then has provided a precise and direct measurement of the top-of-atmosphere GCR flux and its time variation up to $50 \mathrm{GeV} \mathrm{[6];} \mathrm{the} \mathrm{AMS-02} \mathrm{experiment} \mathrm{has} \mathrm{been} \mathrm{installed} \mathrm{in} 2011$ on the International Space Station and recently published the proton flux up to $2 \mathrm{TeV}$, integrated in 3 years, with an error at the \% level [5], which provides the most accurate measurement of the high energy part of the LIS.

During the years, many LIS models have been proposed, all based on fits to data from balloon experiments and spacecrafts or numerical simulations (e.g. GALPROP). The lack of a low energy direct measurement of the LIS has always been a source of uncertainty for the extraction of the solar modulation parameter and the tuning of heliospheric numerical propagation models. Luckily, in 2012, the Voyager 1 spacecraft, launched in 1977, crossed the heliopause and entered interstel- 
lar space [7]: since then, the GCR flux measured by Voyager 1 has remained steady ${ }^{1}$, thereby confirming that what is being observed is actually a LIS. The availability of the high energy proton flux from AMS-02 and the low energy proton flux from Voyager 1 represents an important progress towards the reduction of the uncertainty on the LIS shape, enabling a more accurate determination of the solar modulation parameter and improving the understanding of the GCRs propagation in the heliosphere.

Exploiting these two datasets, a new parametrization for the proton LIS, along with its uncertainty, will be provided. This new LIS model, modulated with the FFA, will be used to fit the monthly proton fluxes measured by PAMELA and the extracted $\phi$ will be compared with the one derived from NMs.

\section{A new parametrization for the proton LIS}

The latest proton fluxes measured by Voyager 1 and AMS-02 constitute an important step in the knowledge of the proton LIS: Voyager 1 observed for the first time the unmodulated spectrum between $\approx 80$ and $\approx 800 \mathrm{MV}$, while AMS- 02 clarified the issue of the rigidity break around 300 GV previously hinted by PAMELA [4]. Together, these two measurements give a precise picture of the proton LIS at very low and very high energy.

Fig. 1 shows the ratio of various LIS models to the BPH00 model (see [8] and references therein) along with the ratio of Voyager 1 [7] and AMS-02 [5] proton fluxes to the same model: it is evident that the models have different shapes at low energy and different spectral index at high energy; more important, all these models have a single power law behavior at high energy, while AMS-02 observed a clear change of slope. These discrepancies compel us to find a new LIS parametrization.

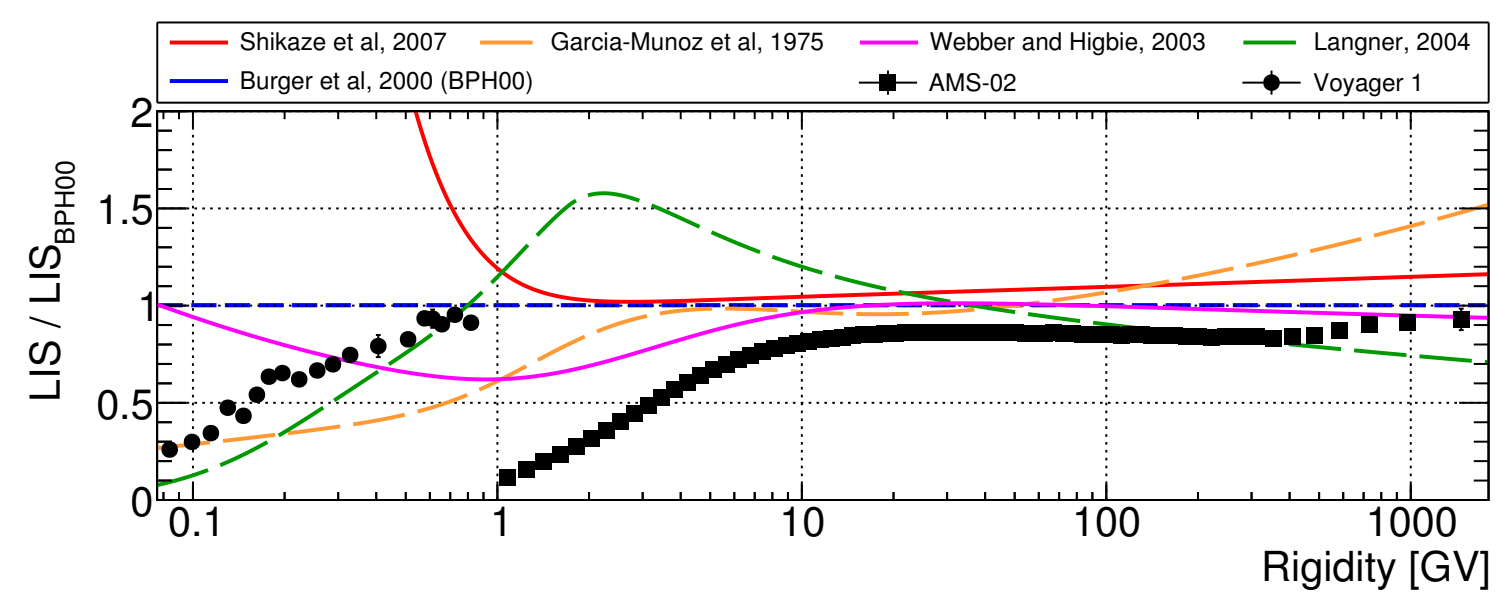

Figure 1: Ratio of various LIS models and Voyager 1 and AMS-02 proton fluxes to BPH00 model.

We will assume that the full LIS can be separated in a product of a high energy part and a low energy part contribution: $J_{L I S}(R)=J_{L I S, L}(R) \cdot J_{L I S, H}(R)$.

\footnotetext{
${ }^{1}$ See, for example, the proton rates from 2013 to 2015: http://voyager.gsfc.nasa.gov/heliopause/yearplot24h.html
} 
For the high energy part of the LIS we will use the same model adopted by the AMS-02 collaboration in their proton flux paper, which describes a continuous change of slope:

$$
J_{L I S, H}(R)=N\left(\frac{R}{45 \mathrm{GV}}\right)^{\gamma}\left[1+\left(\frac{R}{R_{0}}\right)^{\Delta \gamma / s}\right]^{s}
$$

where $\Delta \gamma$ is the change in slope, $R_{0}$ is the rigidity where the two power laws cross and $s$ determines the smoothness of the change ( $s=0$ means a broken power law). It is interesting to note that even fitting above $45 \mathrm{GV}$, the AMS-02 collaboration finds a residual effect from solar modulation: in fact, the error on the normalization $N$ and the spectral index $\gamma$ induced by varying $\phi$ in the fit is as important as the systematic error due to the unfolding and the absolute rigidity scale.

Fig. 2 left shows the ratio of Voyager 1 data to $J_{L I S, H}(R)$. The shape of Voyager 1 ratio suggests a sigmoid function in log scale: we will assume for $J_{L I S, L}$ the following parametrization:

$$
J_{L I S, L}(R)=\left[1+\exp \left(-\frac{\ln R-\mu}{\sigma}\right)\right]^{-1 / v}
$$

where $\mu$ corresponds to the rigidity at which $J_{L I S, L}=1 / 2, \sigma$ determines the steepness of $J_{L I S, L}$ and $v$ describes a possible asymmetry of $J_{L I S, L}$ with respect to $\ln R=\mu$.
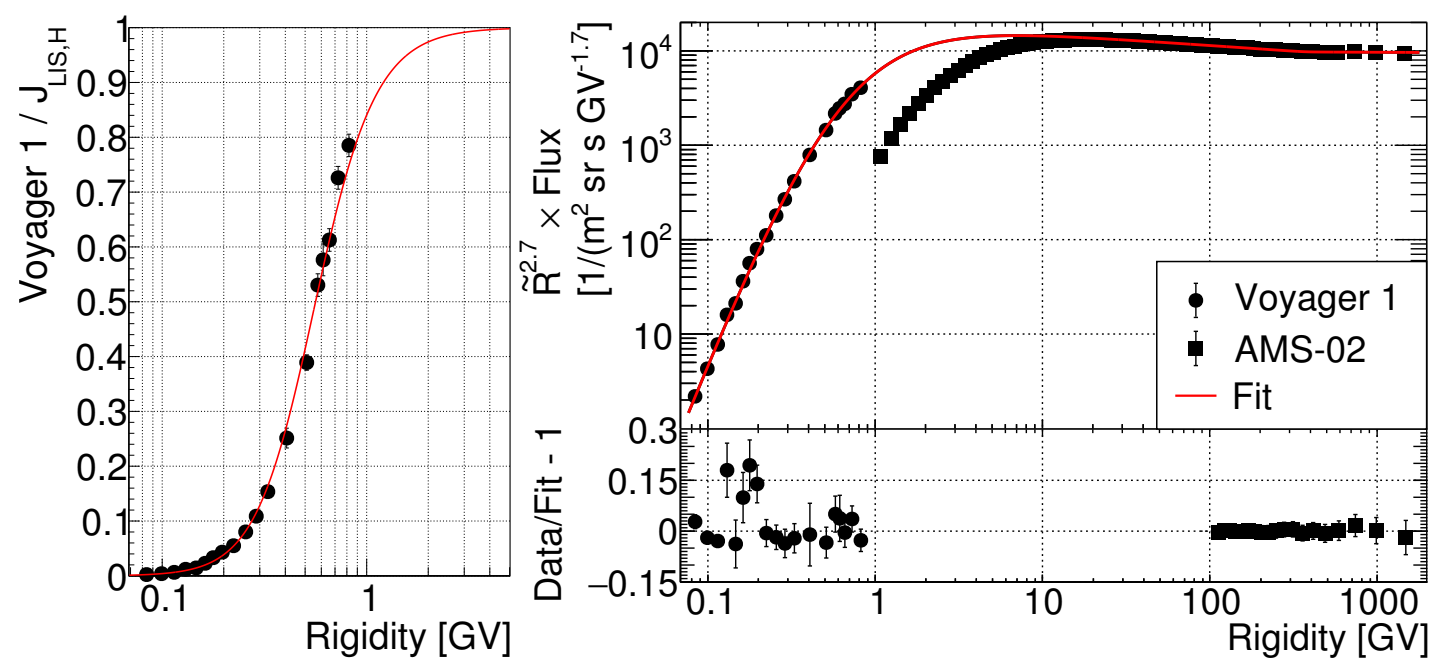

Figure 2: (Left) $J_{L I S, L}$ (red line) and ratio of Voyager 1 proton flux to $J_{L I S, H}$ (black dot). (Right Top) Combined fit of Voyager 1 (black dots) and AMS-02 (black squares) with $J_{L I S}$ (red line); only AMS-02 data points above $100 \mathrm{GV}$ have been included in the fit. (Right Bottom) Fit residuals.

A combined fit of Voyager 1 and AMS-02 data with $J_{L I S}$ has been performed, leaving all the parameters free; the result is shown in Fig. 2 right. For AMS-02, only data points above $100 \mathrm{GV}$ are fitted, so to discard any residual solar modulation (in fact, $\phi=0$ in the fit). In addition, the systematic effect due to the AMS-02 absolute rigidity scale is treated separately, shifting $1 / R$ in the model by $\pm 1 / 26 \mathrm{TV}^{-1}$ (see [5], pag. 5); the treatment of the systematic due to the unfolding requires the knowledge of the AMS-02 resolution matrix, which has not been published, and so 
it is included directly in the data point error. The result has been cross-checked by varying the minimum rigidity of the AMS-02 data points included in the fit between $45 \mathrm{GV}$ and $120 \mathrm{GV}$ : the difference is negligible.

Fig. 3 shows the total LIS relative uncertainty, which is the sum in quadrature of the uncertainty computed propagating the error on the parameters obtained by the fit and the uncertainty of the AMS-02 absolute rigidity scale. The uncertainty is less than $2 \%$ for most of the rigidities, except for the region in which AMS-02 data are not included in the fit, where the uncertainty reach $\approx 5 \%$, and above $400 \mathrm{GV}$, where the error coming from the uncertainty on the AMS-02 absolute rigidity scale dominates.

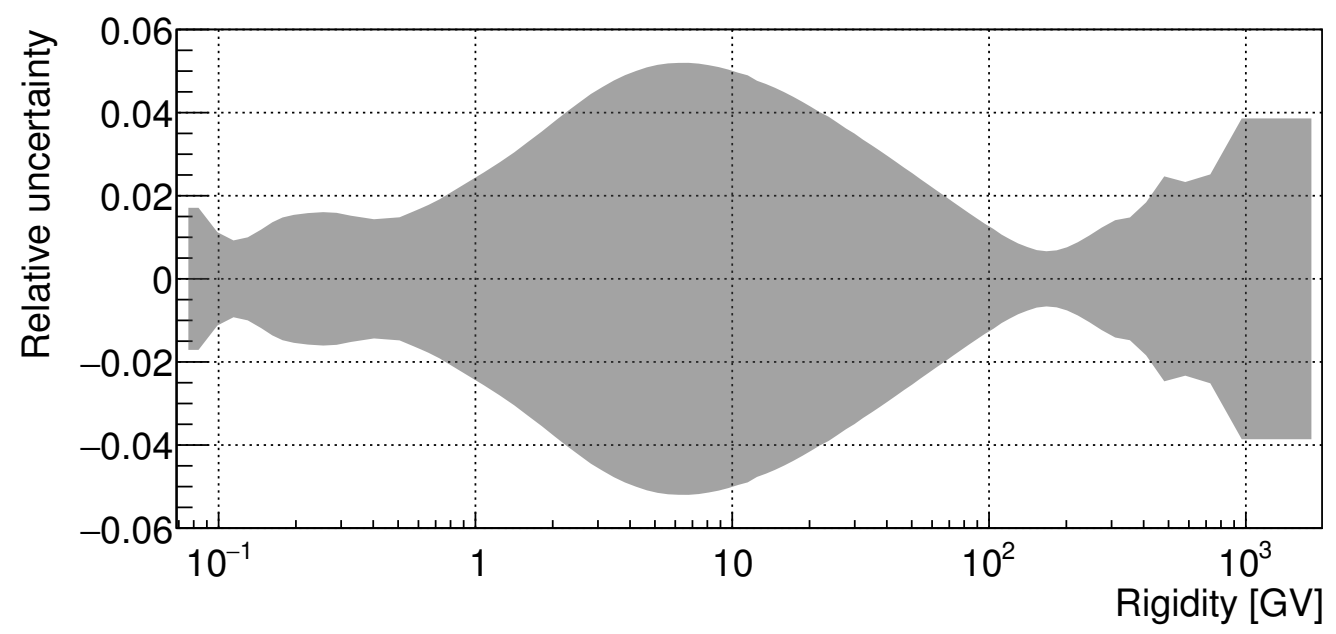

Figure 3: Relative uncertainty on the fitted $J_{L I S}$.

\section{The solar modulation parameter from PAMELA monthly fluxes}

The PAMELA experiment published the proton flux between $0.4 \mathrm{GV}$ and $50 \mathrm{GV}$, integrated in Carrington rotation periods from July 2006 to January 2010 [6]. This dataset provides valuable information to understand the impact of the solar modulation on the differential flux.

Using the force-field approximation from Eq. 1.1, we can fit the PAMELA data with the LIS obtained in the previous section.

Fig. 4 illustrates an example of the fit results (Dec. 20, 2008 - Jan. 11, 2009): the top part shows the PAMELA proton flux (black dots) and the fitted LIS (red line) rescaled by $\widetilde{R}^{2.7}$ (see [9] for the definition of $\widetilde{R}$ ); the bottom part shows the fit residuals (black dots), while the red band represents the systematic uncertainty due to the LIS parametrization.

The systematic error on the fit due to the uncertainty on the LIS has been estimated in the following way: for each Carrington rotation, the fit has been repeated by adding and subtracting to the LIS the relative uncertainty shown in Fig. 3. The uncertainty on $\phi$ coming from the PAMELA fit is $\approx 1.5 \%$, while the one due to the LIS parametrization is $\approx 5 \%$. 


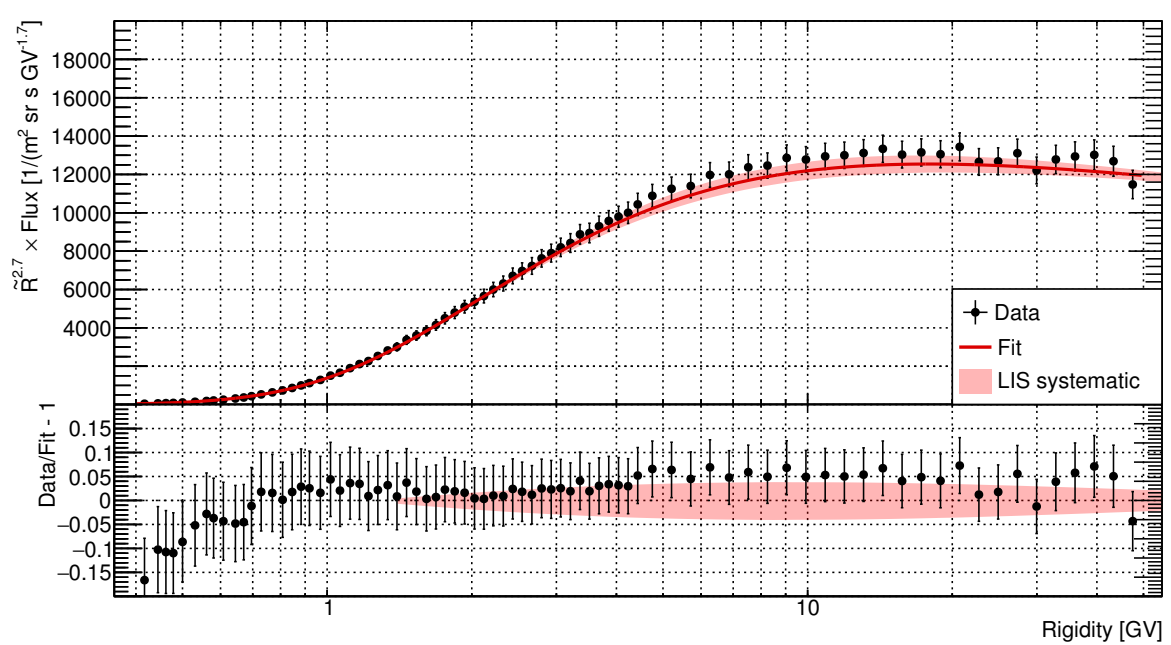

Figure 4: Example of a fit of PAMELA proton flux (Dec. 20, 2008 - Jan. 11, 2009) with Eq. 1.1: on the top, the flux (black dots), the fitted LIS (red line) and the systematic uncertainty due to the LIS parametrization (pink band) rescaled by $\widetilde{R}^{2.7}$; on the bottom, the residuals (black dots) from the fit and the systematic uncertainty due to the LIS parametrization (pink band).

\section{Comparison with neutron monitors}

The solar modulation parameter obtained from the fits to the PAMELA proton fluxes ( $\left.\phi_{P A M E L A}\right)$ is compared to the one derived from the NMs $\left(\phi_{N M}\right)$ [10] in Fig. 5: $\phi_{P A M E L A}$ is always greater than $\phi_{N M}$ by $\approx 50 \mathrm{MV}$. This is due to the fact that there is a dependence of $\phi$ from the LIS used in Eq. 1.1 .

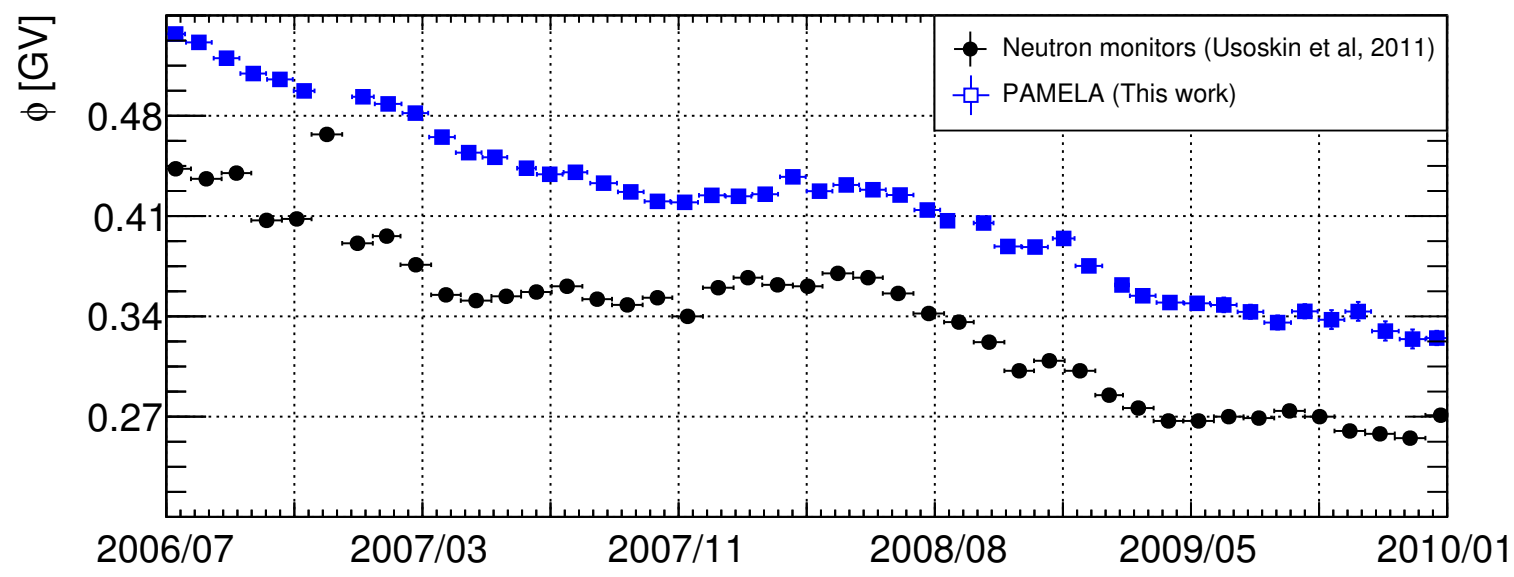

Figure 5: Solar modulation parameter extracted from PAMELA monthly proton fluxes compared with the one derived from neutron monitors.

Fig. 6 shows the correlation between the $\phi_{P A M E L A}$ and $\phi_{N M}$; a robust linear fit has been performed, resulting in the following relation between the two solar modulation parameter: 


$$
\phi_{N M}=0.91 \phi_{P A M E L A}-44 \mathrm{MV}
$$

where $\phi_{N M}$ is derived using as LIS the BPH00 model, while $\phi_{P A M E L A}$ is extracted using the LIS presented in this work.

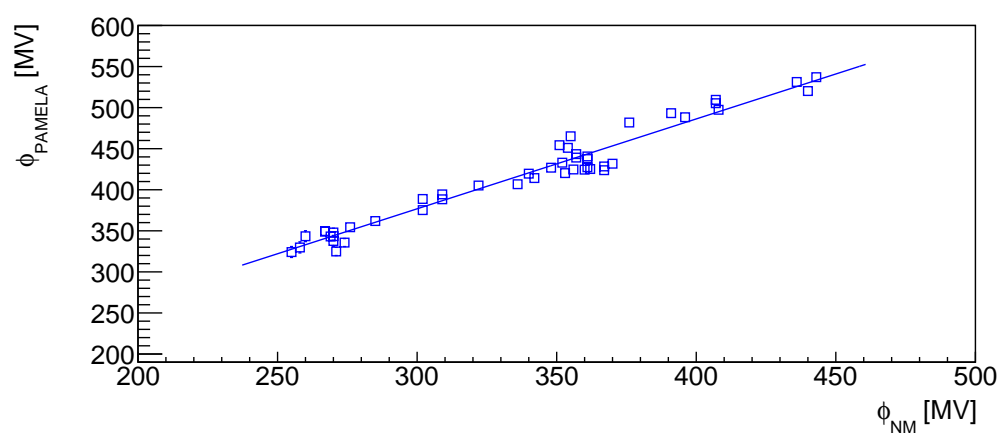

Figure 6: Correlation between $\phi_{P A M E L A}$ and $\phi_{N M}$. The blue line represents a robust linear fit.

\section{Conclusions}

The measurements of the proton flux by Voyager 1 and AMS-02 allows to determine the shape of the LIS more precisely with respect to past: in fact, all previous models do not fit both Voyager 1 and AMS-02 data. A new parametrization of the proton LIS from $80 \mathrm{MV}$ up to $2 \mathrm{TV}$ has been derived in this work: the total uncertainty on this LIS is $2 \%-5 \%$. Using the force-field approximation, this new LIS has been fitted to the monthly proton flux measured by PAMELA in order to extract the solar modulation parameter $\phi$. The total uncertainty on $\phi$ due to the fit and the LIS parametrization is less than $5 \%$.

The solar modulation parameter obtained from PAMELA has been compared with the one derived from the neutron monitors. The difference between them is due to the fact that NM count rates in [10] has been fitted with a different LIS model: an updated analysis of NM data with the LIS presented in this work is ongoing. The relation between the $\phi$ computed in this work and the one provided by NM is given.

\section{References}

[1] E.N. Parker, 1958, Dynamics of the interplanetary gas and magnetic fields, The Astrophysical Journal 128.

[2] L.J. Gleeson and W.I. Axford, 1968, Solar modulation of galactic cosmic rays, The Astrophysical Journal 154.

[3] Y. Shikaze et al., 2007, Measurements of 0.2-20 GeV/n cosmic-ray proton and helium spectra from 1997 through 2002 with the BESS spectrometer, Journal of Astroparticle Physics 28 (1).

[4] O. Adriani et al., 2011, PAMELA measurements of cosmic-ray proton and helium spectra, Science 332 (69). 
[5] M. Aguilar et al., 2015, Precision measurement of the proton flux in primary cosmic rays from rigidity 1 GV to 1.8 TV with the Alpha Magnetic Spectrometer on the International Space Station, Physical Review Letter 114 (171103).

[6] O. Adriani et al., 2013, Time dependence of the proton flux measured by PAMELA during the 2006 July-2009 December solar minimum, The Astrophysical Journal 765 (91).

[7] E. Stone et al., 2013, Voyager 1 observes low-energy galactic cosmic rays in a region depleted of heliospheric ions, Science 341 (150).

[8] I.G. Usoskin et al., 2005, Heliospheric modulation of cosmic rays: Monthly reconstruction for 1951-2004, Journal of Geophysical Research 110 (A12108).

[9] G.D. Lafferty and T.R. Wyatt, 1994, Where to stick your data points: the treatment of measurements within wide bins, NIM A 355 (2-3).

[10] I.G. Usoskin et al., 2011 Solar modulation parameter for cosmic rays since 1936 reconstructed from ground-based neutron monitors and ionization chambers, Journal of Geophysical Research 116 (A02104). 